BABAR-CONF-01/03

SLAC-PUB-8925

hep-ex/0107057

July, 2001

\title{
Measurement of the branching fraction for the decay $B^{0} \rightarrow D^{*+} D^{*-}$
}

\author{
The BABAR Collaboration
}

July 21, 2001

\begin{abstract}
Decays of the type $B \rightarrow D^{(*)} \bar{D}^{(*)}$ can be used to provide a measurement of the parameter $\sin 2 \beta$ of the Unitarity Triangle that is complementary to the measurement derived from the mode $B^{0} \rightarrow$ $J / \psi K_{S}^{0}$. In this document we report a measurement of the branching fraction for the decay $B^{0} \rightarrow$ $D^{*+} D^{*-}$ with the BABAR detector. With data corresponding to an integrated luminosity of $20.7 \mathrm{fb}^{-1}$ collected at the $\Upsilon(4 S)$ resonance during 1999-2000, we have reconstructed 38 candidate signal events in the mode $B^{0} \rightarrow D^{*+} D^{*-}$ with an estimated background of $6.2 \pm 0.5$ events. From these events, we determine the branching fraction to be $\mathcal{B}\left(B^{0} \rightarrow D^{*+} D^{*-}\right)=(8.0 \pm 1.6($ stat $) \pm 1.2($ syst $)) \times$ $10^{-4}$ (preliminary).
\end{abstract}

Submitted to the

$20^{\text {th }}$ International Symposium on Lepton and Photon Interactions at High Energies, $7 / 23-7 / 28 / 2001$, Rome, Italy

Stanford Linear Accelerator Center, Stanford University, Stanford, CA 94309

Work supported in part by Department of Energy contract DE-AC03-76SF00515. 
The BABAR Collaboration,

B. Aubert, D. Boutigny, J.-M. Gaillard, A. Hicheur, Y. Karyotakis, J. P. Lees, P. Robbe, V. Tisserand Laboratoire de Physique des Particules, F-74941 Annecy-le-Vieux, France

A. Palano

Università di Bari, Dipartimento di Fisica and INFN, I-70126 Bari, Italy

G. P. Chen, J. C. Chen, N. D. Qi, G. Rong, P. Wang, Y. S. Zhu

Institute of High Energy Physics, Beijing 100039, China

G. Eigen, P. L. Reinertsen, B. Stugu

University of Bergen, Inst. of Physics, N-5007 Bergen, Norway

B. Abbott, G. S. Abrams, A. W. Borgland, A. B. Breon, D. N. Brown, J. Button-Shafer, R. N. Cahn, A. R. Clark, M. S. Gill, A. V. Gritsan, Y. Groysman, R. G. Jacobsen, R. W. Kadel, J. Kadyk, L. T. Kerth,

S. Kluth, Yu. G. Kolomensky, J. F. Kral, C. LeClerc, M. E. Levi, T. Liu, G. Lynch, A. B. Meyer,

M. Momayezi, P. J. Oddone, A. Perazzo, M. Pripstein, N. A. Roe, A. Romosan, M. T. Ronan, V. G. Shelkov, A. V. Telnov, W. A. Wenzel

Lawrence Berkeley National Laboratory and University of California, Berkeley, CA 94720, USA

P. G. Bright-Thomas, T. J. Harrison, C. M. Hawkes, D. J. Knowles, S. W. O’Neale, R. C. Penny, A. T. Watson, N. K. Watson

University of Birmingham, Birmingham, B15 2TT, United Kingdom

T. Deppermann, K. Goetzen, H. Koch, J. Krug, M. Kunze, B. Lewandowski, K. Peters, H. Schmuecker, M. Steinke

Ruhr Universität Bochum, Institut für Experimentalphysik 1, D-44780 Bochum, Germany

J. C. Andress, N. R. Barlow, W. Bhimji, N. Chevalier, P. J. Clark, W. N. Cottingham, N. De Groot, N. Dyce, B. Foster, J. D. McFall, D. Wallom, F. F. Wilson

University of Bristol, Bristol BS8 1TL, United Kingdom

K. Abe, C. Hearty, T. S. Mattison, J. A. McKenna, D. Thiessen

University of British Columbia, Vancouver, BC, Canada V6T $1 Z 1$

S. Jolly, A. K. McKemey, J. Tinslay

Brunel University, Uxbridge, Middlesex UB8 3PH, United Kingdom

V. E. Blinov, A. D. Bukin, D. A. Bukin, A. R. Buzykaev, V. B. Golubev, V. N. Ivanchenko, A. A. Korol,

E. A. Kravchenko, A. P. Onuchin, A. A. Salnikov, S. I. Serednyakov, Yu. I. Skovpen, V. I. Telnov,

A. N. Yushkov

Budker Institute of Nuclear Physics, Novosibirsk 630090, Russia

D. Best, A. J. Lankford, M. Mandelkern, S. McMahon, D. P. Stoker

University of California at Irvine, Irvine, CA 92697, USA

A. Ahsan, K. Arisaka, C. Buchanan, S. Chun

University of California at Los Angeles, Los Angeles, CA 90024, USA 
J. G. Branson, D. B. MacFarlane, S. Prell, Sh. Rahatlou, G. Raven, V. Sharma

University of California at San Diego, La Jolla, CA 92093, USA

C. Campagnari, B. Dahmes, P. A. Hart, N. Kuznetsova, S. L. Levy, O. Long, A. Lu, J. D. Richman, W. Verkerke, M. Witherell, S. Yellin

University of California at Santa Barbara, Santa Barbara, CA 93106, USA

J. Beringer, D. E. Dorfan, A. M. Eisner, A. Frey, A. A. Grillo, M. Grothe, C. A. Heusch, R. P. Johnson,

W. Kroeger, W. S. Lockman, T. Pulliam, H. Sadrozinski, T. Schalk, R. E. Schmitz, B. A. Schumm,

A. Seiden, M. Turri, W. Walkowiak, D. C. Williams, M. G. Wilson

University of California at Santa Cruz, Institute for Particle Physics, Santa Cruz, CA 95064, USA

E. Chen, G. P. Dubois-Felsmann, A. Dvoretskii, D. G. Hitlin, S. Metzler, J. Oyang, F. C. Porter, A. Ryd, A. Samuel, M. Weaver, S. Yang, R. Y. Zhu

California Institute of Technology, Pasadena, CA 91125, USA

S. Devmal, T. L. Geld, S. Jayatilleke, G. Mancinelli, B. T. Meadows, M. D. Sokoloff

University of Cincinnati, Cincinnati, OH 45221, USA

T. Barillari, P. Bloom, M. O. Dima, S. Fahey, W. T. Ford, D. R. Johnson, U. Nauenberg, A. Olivas, H. Park, P. Rankin, J. Roy, S. Sen, J. G. Smith, W. C. van Hoek, D. L. Wagner

University of Colorado, Boulder, CO 80309, USA

J. Blouw, J. L. Harton, M. Krishnamurthy, A. Soffer, W. H. Toki, R. J. Wilson, J. Zhang

Colorado State University, Fort Collins, CO 80523, USA

T. Brandt, J. Brose, T. Colberg, G. Dahlinger, M. Dickopp, R. S. Dubitzky, A. Hauke, E. Maly,

R. Müller-Pfefferkorn, S. Otto, K. R. Schubert, R. Schwierz, B. Spaan, L. Wilden

Technische Universität Dresden, Institut für Kern- und Teilchenphysik, D-01062, Dresden, Germany

L. Behr, D. Bernard, G. R. Bonneaud, F. Brochard, J. Cohen-Tanugi, S. Ferrag, E. Roussot, S. T'Jampens, Ch. Thiebaux, G. Vasileiadis, M. Verderi

Ecole Polytechnique, F-91128 Palaiseau, France

A. Anjomshoaa, R. Bernet, A. Khan, D. Lavin, F. Muheim, S. Playfer, J. E. Swain

University of Edinburgh, Edinburgh EH9 3JZ, United Kingdom

M. Falbo

Elon University, Elon University, NC 27244-2010, USA

C. Borean, C. Bozzi, S. Dittongo, M. Folegani, L. Piemontese

Università di Ferrara, Dipartimento di Fisica and INFN, I-44100 Ferrara, Italy

E. Treadwell

Florida A $8 M$ University, Tallahassee, FL 3230\%, USA

F. Anulli, ${ }^{1}$ R. Baldini-Ferroli, A. Calcaterra, R. de Sangro, D. Falciai, G. Finocchiaro, P. Patteri,

I. M. Peruzzi, ${ }^{2} 1$ M. Piccolo, Y. Xie, A. Zallo

Laboratori Nazionali di Frascati dell'INFN, I-00044 Frascati, Italy

\footnotetext{
${ }^{1}$ Also with Università di Perugia, I-06100 Perugia, Italy
} 
S. Bagnasco, A. Buzzo, R. Contri, G. Crosetti, P. Fabbricatore, S. Farinon, M. Lo Vetere, M. Macri, M. R. Monge, R. Musenich, M. Pallavicini, R. Parodi, S. Passaggio, F. C. Pastore, C. Patrignani, M. G. Pia, C. Priano, E. Robutti, A. Santroni

Università di Genova, Dipartimento di Fisica and INFN, I-16146 Genova, Italy

M. Morii

Harvard University, Cambridge, MA 02138, USA

R. Bartoldus, T. Dignan, R. Hamilton, U. Mallik

University of Iowa, Iowa City, IA 52242, USA

J. Cochran, H. B. Crawley, P.-A. Fischer, J. Lamsa, W. T. Meyer, E. I. Rosenberg

Iowa State University, Ames, IA 50011-3160, USA

M. Benkebil, G. Grosdidier, C. Hast, A. Höcker, H. M. Lacker, S. Laplace, V. Lepeltier, A. M. Lutz, S. Plaszczynski, M. H. Schune, S. Trincaz-Duvoid, A. Valassi, G. Wormser Laboratoire de l'Accélérateur Linéaire, F-91898 Orsay, France

R. M. Bionta, V. Brigljević, D. J. Lange, M. Mugge, X. Shi, K. van Bibber, T. J. Wenaus, D. M. Wright, C. R. Wuest

Lawrence Livermore National Laboratory, Livermore, CA 94550, USA

M. Carroll, J. R. Fry, E. Gabathuler, R. Gamet, M. George, M. Kay, D. J. Payne, R. J. Sloane, C. Touramanis

University of Liverpool, Liverpool L69 3BX, United Kingdom

M. L. Aspinwall, D. A. Bowerman, P. D. Dauncey, U. Egede, I. Eschrich, N. J. W. Gunawardane, J. A. Nash, P. Sanders, D. Smith

University of London, Imperial College, London, SW7 2BW, United Kingdom

D. E. Azzopardi, J. J. Back, P. Dixon, P. F. Harrison, R. J. L. Potter, H. W. Shorthouse, P. Strother, P. B. Vidal, M. I. Williams

Queen Mary, University of London, E1 4NS, United Kingdom

G. Cowan, S. George, M. G. Green, A. Kurup, C. E. Marker, P. McGrath, T. R. McMahon, S. Ricciardi, F. Salvatore, I. Scott, G. Vaitsas

University of London, Royal Holloway and Bedford New College, Egham, Surrey TW20 0EX, United Kingdom

D. Brown, C. L. Davis

University of Louisville, Louisville, KY 40292, USA

J. Allison, R. J. Barlow, J. T. Boyd, A. C. Forti, J. Fullwood, F. Jackson, G. D. Lafferty, N. Savvas,

E. T. Simopoulos, J. H. Weatherall

University of Manchester, Manchester M13 9PL, United Kingdom

A. Farbin, A. Jawahery, V. Lillard, J. Olsen, D. A. Roberts, J. R. Schieck

University of Maryland, College Park, MD 20742, USA

G. Blaylock, C. Dallapiccola, K. T. Flood, S. S. Hertzbach, R. Kofler, T. B. Moore, H. Staengle, S. Willocq University of Massachusetts, Amherst, MA 01003, USA 
B. Brau, R. Cowan, G. Sciolla, F. Taylor, R. K. Yamamoto

Massachusetts Institute of Technology, Laboratory for Nuclear Science, Cambridge, MA 02139, USA

M. Milek, P. M. Patel, J. Trischuk

McGill University, Montréal, Canada QC H3A 2T8

F. Lanni, F. Palombo

Università di Milano, Dipartimento di Fisica and INFN, I-20133 Milano, Italy

J. M. Bauer, M. Booke, L. Cremaldi, V. Eschenburg, R. Kroeger, J. Reidy, D. A. Sanders, D. J. Summers University of Mississippi, University, MS 38677, USA

J. P. Martin, J. Y. Nief, R. Seitz, P. Taras, A. Woch, V. Zacek

Université de Montréal, Laboratoire René J. A. Lévesque, Montréal, Canada QC H3C 3J7

H. Nicholson, C. S. Sutton

Mount Holyoke College, South Hadley, MA 01075, USA

C. Cartaro, N. Cavallo, ${ }^{3}$ G. De Nardo, F. Fabozzi, C. Gatto, L. Lista, P. Paolucci, D. Piccolo, C. Sciacca Università di Napoli Federico II, Dipartimento di Scienze Fisiche and INFN, I-80126, Napoli, Italy

J. M. LoSecco

University of Notre Dame, Notre Dame, IN 46556, USA

J. R. G. Alsmiller, T. A. Gabriel, T. Handler

Oak Ridge National Laboratory, Oak Ridge, TN 37831, USA

J. Brau, R. Frey, M. Iwasaki, N. B. Sinev, D. Strom

University of Oregon, Eugene, OR 97403, USA

F. Colecchia, F. Dal Corso, A. Dorigo, F. Galeazzi, M. Margoni, G. Michelon, M. Morandin, M. Posocco, M. Rotondo, F. Simonetto, R. Stroili, E. Torassa, C. Voci

Università di Padova, Dipartimento di Fisica and INFN, I-35131 Padova, Italy

M. Benayoun, H. Briand, J. Chauveau, P. David, Ch. de la Vaissière, L. Del Buono, O. Hamon, F. Le Diberder, Ph. Leruste, J. Lory, L. Roos, J. Stark, S. Versillé

Universités Paris VI et VII, Lab de Physique Nucléaire H. E., F-75252 Paris, France

P. F. Manfredi, V. Re, V. Speziali

Università di Pavia, Dipartimento di Elettronica and INFN, I-27100 Pavia, Italy

E. D. Frank, L. Gladney, Q. H. Guo, J. H. Panetta

University of Pennsylvania, Philadelphia, PA 19104, USA

C. Angelini, G. Batignani, S. Bettarini, M. Bondioli, M. Carpinelli, F. Forti, M. A. Giorgi, A. Lusiani, F. Martinez-Vidal, M. Morganti, N. Neri, E. Paoloni, M. Rama, G. Rizzo, F. Sandrelli, G. Simi, G. Triggiani, J. Walsh

Università di Pisa, Scuola Normale Superiore and INFN, I-56010 Pisa, Italy

\footnotetext{
${ }^{3}$ Also with Università della Basilicata, I-85100 Potenza, Italy
} 
M. Haire, D. Judd, K. Paick, L. Turnbull, D. E. Wagoner

Prairie View A\&M University, Prairie View, TX 77446, USA

J. Albert, C. Bula, P. Elmer, C. Lu, K. T. McDonald, V. Miftakov, S. F. Schaffner, A. J. S. Smith, A. Tumanov, E. W. Varnes

Princeton University, Princeton, NJ 08544, USA

G. Cavoto, D. del Re, R. Faccini, ${ }^{4}$ F. Ferrarotto, F. Ferroni, K. Fratini, E. Lamanna, E. Leonardi, M. A. Mazzoni, S. Morganti, G. Piredda, F. Safai Tehrani, M. Serra, C. Voena Università di Roma La Sapienza, Dipartimento di Fisica and INFN, I-00185 Roma, Italy

S. Christ, R. Waldi

Universität Rostock, D-18051 Rostock, Germany

P. F. Jacques, M. Kalelkar, R. J. Plano

Rutgers University, New Brunswick, NJ 08903, USA

T. Adye, B. Franek, N. I. Geddes, G. P. Gopal, S. M. Xella

Rutherford Appleton Laboratory, Chilton, Didcot, Oxon, OX11 0QX, United Kingdom

R. Aleksan, G. De Domenico, S. Emery, A. Gaidot, S. F. Ganzhur, P.-F. Giraud, G. Hamel de Monchenault, W. Kozanecki, M. Langer, G. W. London, B. Mayer, B. Serfass, G. Vasseur, Ch. Yèche, M. Zito

DAPNIA, Commissariat à l'Energie Atomique/Saclay, F-91191 Gif-sur-Yvette, France

N. Copty, M. V. Purohit, H. Singh, F. X. Yumiceva

University of South Carolina, Columbia, SC 29208, USA

I. Adam, P. L. Anthony, D. Aston, K. Baird, J. P. Berger, E. Bloom, A. M. Boyarski, F. Bulos,

G. Calderini, R. Claus, M. R. Convery, D. P. Coupal, D. H. Coward, J. Dorfan, M. Doser, W. Dunwoodie,

R. C. Field, T. Glanzman, G. L. Godfrey, S. J. Gowdy, P. Grosso, T. Himel, T. Hryn'ova, M. E. Huffer,

W. R. Innes, C. P. Jessop, M. H. Kelsey, P. Kim, M. L. Kocian, U. Langenegger, D. W. G. S. Leith, S. Luitz, V. Luth, H. L. Lynch, H. Marsiske, S. Menke, R. Messner, K. C. Moffeit, R. Mount, D. R. Muller, C. P. O'Grady, M. Perl, S. Petrak, H. Quinn, B. N. Ratcliff, S. H. Robertson, L. S. Rochester,

A. Roodman, T. Schietinger, R. H. Schindler, J. Schwiening, V. V. Serbo, A. Snyder, A. Soha, S. M. Spanier, J. Stelzer, D. Su, M. K. Sullivan, H. A. Tanaka, J. Va'vra, S. R. Wagner,

A. J. R. Weinstein, W. J. Wisniewski, D. H. Wright, C. C. Young

Stanford Linear Accelerator Center, Stanford, CA 94309, USA

P. R. Burchat, C. H. Cheng, D. Kirkby, T. I. Meyer, C. Roat

Stanford University, Stanford, CA 94305-4060, USA

R. Henderson

TRIUMF, Vancouver, BC, Canada V6T $2 A 3$

W. Bugg, H. Cohn, A. W. Weidemann

University of Tennessee, Knoxville, TN 37996, USA

\footnotetext{
${ }^{4}$ Also with University of California at San Diego, La Jolla, CA 92093, USA
} 
J. M. Izen, I. Kitayama, X. C. Lou, M. Turcotte

University of Texas at Dallas, Richardson, TX 75083, USA

F. Bianchi, M. Bona, B. Di Girolamo, D. Gamba, A. Smol, D. Zanin

Università di Torino, Dipartimento di Fisica Sperimentale and INFN, I-10125 Torino, Italy

L. Bosisio, G. Della Ricca, L. Lanceri, A. Pompili, P. Poropat, M. Prest, E. Vallazza, G. Vuagnin

Università di Trieste, Dipartimento di Fisica and INFN, I-34127 Trieste, Italy

R. S. Panvini

Vanderbilt University, Nashville, TN 37235, USA

C. M. Brown, A. De Silva, R. Kowalewski, J. M. Roney

University of Victoria, Victoria, BC, Canada V8W $3 P 6$

H. R. Band, E. Charles, S. Dasu, F. Di Lodovico, A. M. Eichenbaum, H. Hu, J. R. Johnson, R. Liu, J. Nielsen, Y. Pan, R. Prepost, I. J. Scott, S. J. Sekula, J. H. von Wimmersperg-Toeller, S. L. Wu, Z. Yu, H. Zobernig

University of Wisconsin, Madison, WI 53706, USA

T. M. B. Kordich, H. Neal

Yale University, New Haven, CT 06511, USA 


\section{Introduction}

One of the most important goals of the BABAR experiment is to precisely measure the angles of the Unitarity Triangle. While the decay $B^{0} \rightarrow J / \psi K_{S}^{0}$ can be used to measure $\sin 2 \beta$, the Standard Model predicts that the time-dependent $C P$ violating asymmetries in the decays [1] $B^{0} \rightarrow D^{(*)+} D^{(*)-}$ can also be used to measure the same quantity. An independent measurement of $\sin 2 \beta$ in these modes would therefore provide a consistency test of $C P$-violation in the Standard Model.

The vector-vector decay $B^{0} \rightarrow D^{*+} D^{*-}$ is not, however, a pure $C P$ eigenstate. A sizeable dilution of the measured asymmetry may be produced by a non-negligible $P$-wave $C P$-odd component. The dilution can, in principle, be completely removed by a time-dependent angular analysis of the decay products $[2]$.

The rate for the Cabibbo-suppressed decays $B \rightarrow D^{(*)} \bar{D}^{(*)}$ can be estimated from the measured rate of the Cabibbo-favored decays $B \rightarrow D_{S}^{(*)} \bar{D}^{(*)}$ :

$$
\mathcal{B}\left(B \rightarrow D^{(*)} \bar{D}^{(*)}\right) \approx\left(\frac{f_{D^{(*)}}}{f_{D_{S}^{(*)}}}\right) \tan ^{2} \theta_{C} \cdot \mathcal{B}\left(B \rightarrow D_{S}^{(*)} \bar{D}^{(*)}\right),
$$

where $\theta_{C}$ is the Cabibbo angle, and $f_{D^{(*)}}$ and $f_{D_{S}^{(*)}}$ are decay constants. From this it follows that the $B \rightarrow D^{(*)} \bar{D}^{(*)}$ branching fractions are of the order of $0.1 \%$. Previous measurements of branching fractions and upper limits for these modes are summarized in Table 1.

Table 1: Summary of branching fraction and upper limit measurements performed by the CLEO experiment [3]. Upper limits are quoted at the $90 \%$ confidence level.

\begin{tabular}{cc}
\hline \hline \multicolumn{1}{c}{ Decay } & Branching Fraction $\left(\times 10^{-4}\right)$ \\
\hline$B^{0} \rightarrow D^{*+} D^{*-}$ & $9.9_{-3.3}^{+4.2}($ stat $) \pm 1.2($ syst $)$ \\
$B^{0} \rightarrow D^{*+} D^{-}$ & $<6.3$ \\
$B^{0} \rightarrow D^{+} D^{-}$ & $<9.4$ \\
\hline \hline
\end{tabular}

\section{The BABAR detector and dataset}

The data used in this analysis were collected with the BABAR detector [4] at the PEP-II storage ring [5] located at the Stanford Linear Accelerator Center. The results presented in this paper are based on data taken during the 1999-2000 run. This data sample represents an integrated luminosity of $23.3 \mathrm{fb}^{-1}$, with $20.7 \mathrm{fb}^{-1}$ collected on the $\Upsilon(4 S)$ resonance. The total number of $B \bar{B}$ pairs produced in this sample was $N_{B \bar{B}}=22.7 \times 10^{6}$.

Charged particles are detected and their momenta measured with the combination of a 40-layer drift chamber $(\mathrm{DCH})$ and a five-layer silicon vertex tracker (SVT) embedded in a $1.5 \mathrm{~T}$ solenoidal magnetic field. Photons are detected by a CsI electromagnetic calorimeter (EMC) that provides excellent angular and energy resolutions with a high efficiency for energies above $20 \mathrm{MeV}$. Charged particle identification is provided by the specific ionization loss $(\mathrm{d} E / \mathrm{d} x)$ in the tracking devices and by an internally reflecting ring-imaging Cherenkov detector (DIRC) covering the barrel region of the detector. 
Table 2: $D^{0}$ and $D^{+}$decay modes and branching fractions [7]. The branching fraction for $K_{S}^{0} \rightarrow$ $\pi^{+} \pi^{-}$is included for modes containing a $K_{S}^{0}$.

\begin{tabular}{lc}
\hline \hline Decay Mode & Branching Fraction (\%) \\
\hline$D^{0} \rightarrow K^{-} \pi^{+}$ & $3.83 \pm 0.09$ \\
$D^{0} \rightarrow K^{-} \pi^{+} \pi^{0}$ & $13.9 \pm 0.9$ \\
$D^{0} \rightarrow K^{-} \pi^{+} \pi^{+} \pi^{-}$ & $7.49 \pm 0.31$ \\
$D^{0} \rightarrow K_{S}^{0} \pi^{+} \pi^{-}$ & $1.85 \pm 0.14$ \\
\hline Total $D^{0}$ Branching Fraction & 27.1 \\
\hline \hline Decay Mode & Branching Fraction (\%) \\
\hline$D^{+} \rightarrow K^{-} \pi^{+} \pi^{+}$ & $9.0 \pm 0.6$ \\
$D^{+} \rightarrow K_{S}^{0} \pi^{+}$ & $0.99 \pm 0.09$ \\
$D^{+} \rightarrow K^{-} K^{+} \pi^{+}$ & $0.87 \pm 0.07$ \\
\hline Total $D^{+}$Branching Fraction & 10.9 \\
\hline \hline
\end{tabular}

\section{Determination of $\mathcal{B}\left(B^{0} \rightarrow D^{*+} D^{*-}\right)$}

$B^{0}$ mesons are exclusively reconstructed by combining two charged $D^{*}$ candidates reconstructed in a number of $D^{*}$ and $D$ decay modes. Events are pre-selected by requiring that there be three or more charged tracks and that the normalized second Fox-Wolfram moment [6] of the event be less than 0.6. We also require that the cosine of the angle between the reconstructed $B$ direction and the thrust axis of the rest of the event be less than 0.9 .

Charged kaon candidates are required to be inconsistent with the pion hypothesis, as inferred from the Cherenkov ring measured by the DIRC and the $\mathrm{d} E / \mathrm{d} x$ as measured by the SVT and DCH. There are two exceptions to this: tighter kaon identification is applied to one of the charged kaons in decay $D^{+} \rightarrow K^{-} K^{+} \pi^{+}$, and no particle identification requirements are made for the kaon from the decay $D^{0} \rightarrow K^{-} \pi^{+}$.

$K_{S}^{0} \rightarrow \pi^{+} \pi^{-}$candidates are required to have an invariant mass within $25 \mathrm{MeV} / c^{2}$ of the nominal $K_{S}^{0}$ mass. The opening angle between the flight direction and the momentum vector of the $K_{S}^{0}$ candidate is required to be less than $200 \mathrm{mrad}$, and the transverse flight distance from the primary event vertex must be greater than $2 \mathrm{~mm}$.

Neutral pion candidates are formed from pairs of photons in the EMC with energy above $30 \mathrm{MeV}$, an invariant mass within $20 \mathrm{MeV} / c^{2}$ of the nominal $\pi^{0}$ mass, and a summed energy greater than $200 \mathrm{MeV}$. A mass-constraint fit is then applied to these $\pi^{0}$ candidates. The $\pi^{0}$ from $D^{*+} \rightarrow D^{+} \pi^{0}$ decays ("soft" $\pi^{0}$ ), however, is required to have an invariant mass within $35 \mathrm{MeV} / c^{2}$ of the nominal $\pi^{0}$ mass and momentum in the $\Upsilon(4 S)$ frame of $70<p^{*}<450 \mathrm{MeV} / c$, with no requirement on the summed photon energy.

The decay modes of the $D^{0}$ and $D^{+}$used in this analysis were selected by an optimization of $S^{2} /(S+B)$ based on Monte Carlo simulations, where $S$ is the expected number of signal events and $B$ is the expected number of background events. The $D^{0}$ and $D^{+}$modes used and their branching fractions are summarized in Table 2. $D^{0}\left(D^{+}\right)$meson candidates are required to have an invariant mass within $20 \mathrm{MeV} / c^{2}$ of the nominal $D^{0}\left(D^{+}\right)$mass. 
Table 3: $D^{*}$ decay modes and branching fractions [7].

\begin{tabular}{lcc}
\hline \hline Particle & Decay Mode & Branching Fraction (\%) \\
\hline$D^{*+}$ & $D^{*+} \rightarrow D^{0} \pi^{+}$ & $67.7 \pm 0.5$ \\
& $D^{*+} \rightarrow D^{+} \pi^{0}$ & $30.7 \pm 0.5$ \\
Total Visible $D^{*+}$ Branching Fraction & & 98.4 \\
\hline \hline
\end{tabular}

The $D^{*+}$ mesons are reconstructed in their decays $D^{*+} \rightarrow D^{0} \pi^{+}$and $D^{*+} \rightarrow D^{+} \pi^{0}$. We include for this analysis the decay combinations $D^{*+} D^{*-}$ decaying to $\left(D^{0} \pi^{+}, \bar{D}^{0} \pi^{-}\right)$or $\left(D^{0} \pi^{+}, D^{-} \pi^{0}\right)$, but not $\left(D^{+} \pi^{0}, D^{-} \pi^{0}\right)$ due to the smaller branching fraction and larger expected backgrounds. The branching fractions for these modes are summarized in Table $3 . D^{0}$ and $D^{+}$candidates are subjected to a mass-constraint fit and then combined with soft pion candidates. A vertex fit is performed that includes the position of the beam spot to improve the angular resolution of the soft pion.

To select $B^{0}$ candidates with well reconstructed $D^{*}$ and $D$ mesons, we construct a $\chi^{2}$ that includes all measured $D^{*}$ and $D$ masses:

$$
\begin{aligned}
& \chi_{\text {Mass }}^{2}=\left(\frac{m_{D}-m_{D_{P D G}}}{\sigma_{m_{D}}}\right)^{2}+\left(\frac{m_{\bar{D}}-m_{\bar{D}_{P D G}}}{\sigma_{m_{\bar{D}}}}\right)^{2} \\
&+\left(\frac{\Delta m_{D^{*}}-\Delta m_{D_{P D G}^{*}}}{\sigma_{\Delta m}}\right)^{2}+\left(\frac{\Delta m_{\bar{D}^{*}}-\Delta m_{D_{P D G}^{*}}}{\sigma_{\Delta m}}\right)^{2}
\end{aligned}
$$

where the subscript $P D G$ refers to the nominal value, and $\Delta m$ is the $D^{*}-D$ mass difference. For $\sigma_{m_{D}}$ we use values computed for each $D$ candidate, while for $\sigma_{\Delta m}$ we use fixed values of $0.83 \mathrm{MeV} / c^{2}$ for $D^{*+} \rightarrow D^{0} \pi^{+}$and $1.18 \mathrm{MeV} / c^{2}$ for $D^{*+} \rightarrow D^{+} \pi^{0}$. A requirement that $\chi_{\text {Mass }}^{2}<20$ is applied to all $B^{0}$ candidates. In events with more than one $B^{0}$ candidate, we chose the candidate with the lowest value of $\chi_{\text {Mass }}^{2}$.

A $B$ meson candidate is characterized by two kinematic variables. We use the energy-substituted mass, $m_{\mathrm{ES}}$, defined as

$$
m_{\mathrm{ES}} \equiv \sqrt{E_{\text {Beam }}^{* 2}-p_{B}^{* 2}}
$$

and the difference of the $B$ candidate's energy from the beam energy, $\Delta E$,

$$
\Delta E \equiv E_{B}^{*}-E_{B e a m}^{*}
$$

where $E_{B}^{*}\left(p_{B}^{*}\right)$ are the energy (momentum) of the $B$ candidate in the center-of-mass frame and $E_{\text {Beam }}^{*}$ is one-half of the center-of-mass energy. The signal region in the $\Delta E v s . m_{\text {ES }}$ plane is defined to be $|\Delta E|<25 \mathrm{MeV}$ and $5.273<m_{\mathrm{ES}}<5.285 \mathrm{GeV} / c^{2}$. The width of this region corresponds to approximately $\pm 2.5 \sigma$ in both $\Delta E$ and $m_{\mathrm{ES}}$.

To estimate the contribution from background in the signal region, we define a sideband in the $\Delta E$ vs. $m_{\mathrm{ES}}$ plane as

$$
\begin{gathered}
|\Delta E|<200 \mathrm{MeV} \\
5.20<m_{\mathrm{ES}}<5.26 \mathrm{GeV} / c^{2}
\end{gathered}
$$


and

$$
\begin{gathered}
50<|\Delta E|<200 \mathrm{MeV} \\
5.26<m_{\mathrm{ES}}<5.29 \mathrm{GeV} / c^{2}
\end{gathered}
$$

We parameterize the shape of the background in the $\Delta E$ vs. $m_{\mathrm{ES}}$ plane as the product of an ARGUS function [8] in $m_{\mathrm{ES}}$ and a first-order polynomial in $\Delta E$. Based on this parameterization we estimate that the ratio of the number of background events in the signal region to the number in the sideband region is $(1.72 \pm 0.10) \times 10^{-2}$. The uncertainty is derived from the observed variation of this ratio under alternative assumptions for the background shape in $m_{\mathrm{ES}}$ and $\Delta E$.

Figure 1 shows the events in the $\Delta E v s . m_{\mathrm{ES}}$ plane after all selection criteria have been applied. The small box in the figure indicates the signal region defined above, and the sideband is the entire plane excluding the region bounded by the larger box outside the signal region. There are a total of 38 events located in the signal region, with 363 events in the sideband region. The latter, together with the effective ratio of areas of the signal region to the sideband region, implies an expected number of background events in the signal region of $6.24 \pm 0.33$ (stat) \pm 0.36 (syst). The quoted systematic uncertainty comes from the background shape variation discussed previously. Figure 2 shows a projection of the data on to the $m_{\mathrm{ES}}$ axis after requiring $|\Delta E|<25 \mathrm{MeV}$.

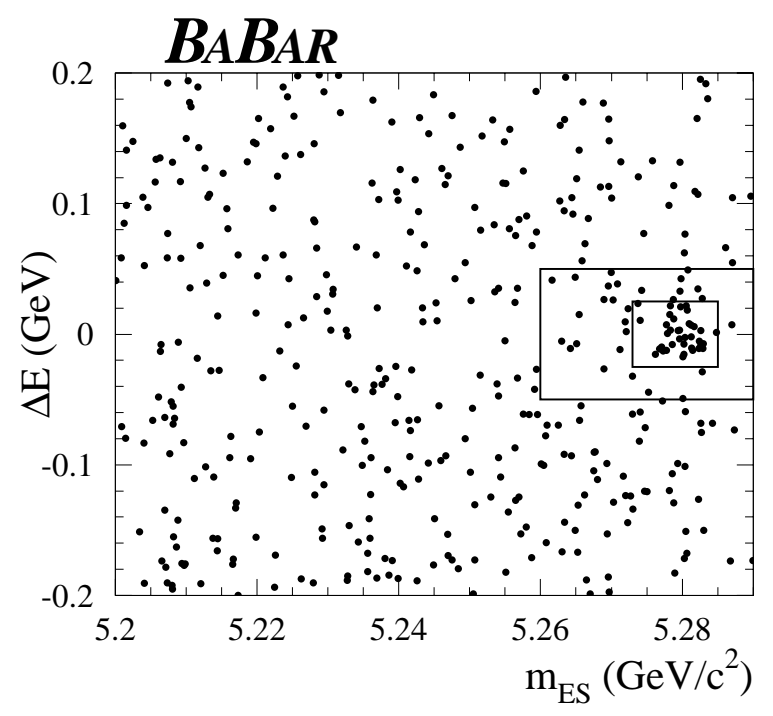

Figure 1: Distribution of events in the $\Delta E$ vs. $m_{\mathrm{ES}}$ plane. The small box indicates the signal region, while the sideband region is everything outside the larger box.

We use a detailed Monte Carlo simulation of the BABAR detector to determine the efficiency for reconstructing the signal. This, together with the total number of $B \bar{B}$ pairs produced during data collection, allows us to determine a preliminary branching fraction for $B^{0} \rightarrow D^{*+} D^{*-}$ to be

$$
\mathcal{B}\left(B^{0} \rightarrow D^{*+} D^{*-}\right)=(8.0 \pm 1.6(\text { stat }) \pm 1.2(\text { syst })) \times 10^{-4}
$$

The dominant systematic uncertainty in this measurement comes from our level of understanding of the charged particle tracking efficiency (9.4\%). The high charged particle multiplicity in this decay mode makes this measurement particularly sensitive to tracking efficiency. Uncertainties were assigned on a per track basis for $\pi, K$ and slow $\pi$, and were added linearly due to large 


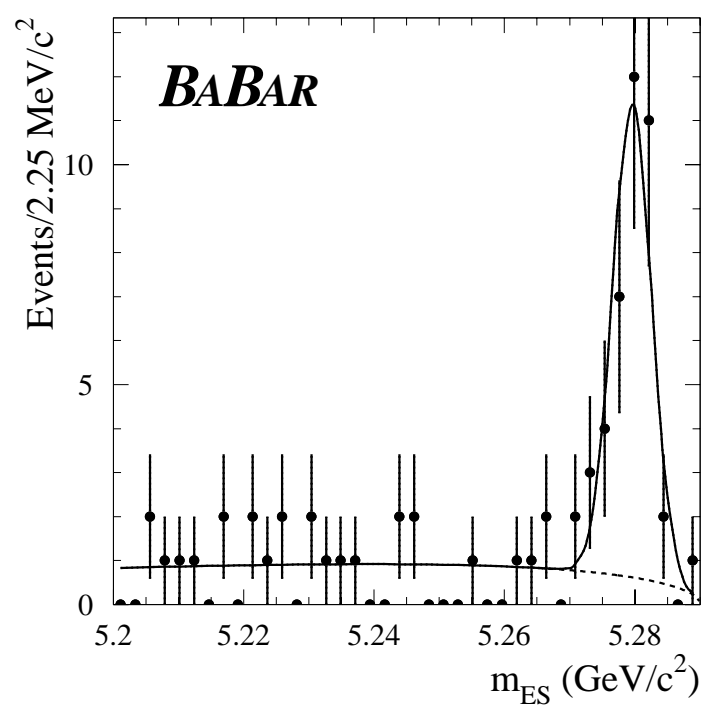

Figure 2: Distribution of events in $m_{\mathrm{ES}}$ plane with a cut of $|\Delta E|<25 \mathrm{MeV}$ applied. The curve represents a fit to the distribution of the sum of a Gaussian to model the signal and an ARGUS function [8] to model the background shape.

correlations. The imprecisely known partial-wave content of the $B^{0} \rightarrow D^{*+} D^{*-}$ final state is another source of systematic uncertainty $(6.6 \%)$. This was estimated by calculating the change in the reconstruction efficiency for different final angular states in Monte Carlo. Other significant systematic uncertainties arise due to the uncertainties on the $D^{*+}, D^{0}$ and $D^{+}$branching fractions $(5.6 \%)$ and the differences in mass resolutions between Monte Carlo and data (4.1\%). The total systematic uncertainty from all sources is $14.5 \%$.

\section{Summary}

Using data collected by the BABAR experiment during 1999-2000, we have observed a signal of $31.8 \pm 6.2($ stat $) \pm 0.4$ (syst) events in the decay $B^{0} \rightarrow D^{*+} D^{*-}$. We measure a preliminary branching ratio to be

$$
\mathcal{B}\left(B^{0} \rightarrow D^{*+} D^{*-}\right)=(8.0 \pm 1.6(\text { stat }) \pm 1.2(\text { syst })) \times 10^{-4}
$$

\section{Acknowledgments}

We are grateful for the extraordinary contributions of our PEP-II colleagues in achieving the excellent luminosity and machine conditions that have made this work possible. The collaborating institutions wish to thank SLAC for its support and the kind hospitality extended to them. This work is supported by the US Department of Energy and National Science Foundation, the Natural Sciences and Engineering Research Council (Canada), Institute of High Energy Physics (China), the Commissariat à l'Energie Atomique and Institut National de Physique Nucléaire et de Physique des Particules (France), the Bundesministerium für Bildung und Forschung (Germany), the Istituto Nazionale di Fisica Nucleare (Italy), the Research Council of Norway, the Ministry of Science and Technology of the Russian Federation, and the Particle Physics and Astronomy Research Council 
(United Kingdom). Individuals have received support from the Swiss National Science Foundation, the A. P. Sloan Foundation, the Research Corporation, and the Alexander von Humboldt Foundation.

\section{References}

[1] Charge-conjugate states are implied throughout this paper and the symbol $D^{(*)}$ refers to either $D$ or $D^{*}$.

[2] I. Dunietz et al., Phys. Rev. D 43, 2193 (1991).

[3] E. Lipeles et al., Phys. Rev. D 62, 032005 (2000).

[4] The BABAR Collaboration, B. Aubert et al., SLAC-PUB-8596, to appear in Nucl. Instrum. Methods.

[5] PEP-II Conceptual Design Report, SLAC-R-418 (1993).

[6] G. C. Fox and S. Wolfram, Phys. Rev. Lett. 41, 1581 (1978).

[7] Particle Data Group, D. E. Groom et al., Eur. Phys. Jour. C 15, 1 (2000).

[8] ARGUS Collaboration, H. Albrecht et al., Phys. Lett. B185, 218 (1987). 